\title{
Failure strength prediction of glass/epoxy composite laminates from acoustic emission parameters using artificial neural network
}

\author{
C. Suresh Kumar ${ }^{\text {a }}$, V. Arumugam ${ }^{a}$, R. Sengottuvelusamy ${ }^{\mathrm{b}}$, S. Srinivasan ${ }^{\mathrm{b}}$, H.N. Dhakal ${ }^{\text {c* }}$ \\ ${ }^{a}$ Department of Aerospace Engineering, MIT campus, Chromepet, Anna University, \\ Chennai-44,TamilNadu, India \\ ${ }^{b}$ Department of Instrumentation Engineering, MIT campus, Chromepet, Anna University, \\ Chennai-44,TamilNadu, India \\ ${ }^{c}$ Advanced Polymer and Composites (APC) Research Group, School of Engineering, \\ University of Portsmouth, Anglesea Road, Anglesea Building, Portsmouth, Hampshire PO1
} $3 D J, U K$

\begin{abstract}
:
The ageing effect of glass/epoxy composite laminates exposed to seawater environment for different periods of time was investigated using acoustic emission (AE) monitoring. The mass gain ratio and flexural strength of glass fiber reinforced plastic (GFRP) composite laminates were examined after the seawater treatment. The flexural strength of the seawater treated GFRP specimens showed a decreasing trend with increasing exposure time. The degradation effects of seawater are studied based on the changes in AE signal parameters for various periods of time. The significant $\mathrm{AE}$ parameters like counts, energy, signal strength, absolute energy and hits were considered as training data input. The input data were taken from $40 \%$ to $70 \%$ of failure loads for developing the radial basis function neural network (RBFNN) and generalised regression neural network (GRNN) models. RBFNN model was able to predict the ultimate failure strength and could be validated with the experimental results with the percentage error well within $0.5 \%-7.2 \%$ tolerance, whereas GRNN model was able to predict the ultimate failure strength with the percentage error well within $0.5 \%-4.4 \%$ tolerance. The prediction accuracy of GRNN model is found to be better than RBFNN model.
\end{abstract}

Keywords: GFRP composite laminates, Acoustic emission, Seawater degradation, Artificial neural network, RBFNN, GRNN.

* Corresponding author. Tel: + 44 (0) 239284 2582; fax: + 44 (0) 2392842351.

E-mail: hom.dhakal@port.ac.uk 


\section{Introduction}

Glass fiber reinforced polymer (GFRP) composite materials are widely used in marine, aircraft, spacecraft and automobile engineering due to their high strength to weight ratio. However, wet environments greatly influence the strength of composite materials. The mechanical properties of composite material become weaker and induced degradation on exposure to seawater. The degradation property of GFRP is unavoidable in marine applications; the laminates may be corroded after long-term service in seawater environment $[1,2]$. The most noticeable effects of exposure to moisture are the plasticization of matrix and degradation of fiber matrix interface [3-5]. The structural health monitoring (SHM) of composite materials is important in seawater environment, due to degradation induced during in-service operation resulting in failure of composite structure. Hence, artificial neural network (ANN) works well in predicting the ultimate failure strength of composite materials. ANN was developed especially to relate the mechanical parameters of the material between the experimental and simulated values. ANN model simulates different mechanical behaviours of composite materials related to non-linear properties including elasticity, plasticity, viscoelasticity [6]. Perera et al [7] found that ANN model successfully simulated the desired phenomenon and the prediction accuracy was improved by increasing the training database.

When a material is strained, various failure modes are activated such that, a part of the total strain energy is dissipated as a wave that propagates from the failure source through the medium [8]. The resulting stress waves are acoustic emissions (AE) which are transient elastic waves generated by rapid release of energy from localized sources within a material when it undergoes deformation [9]. The significant AE parameters such as rise time, count, energy, duration and amplitude were used for materials characterisation and structural integrity evaluation [10-13]. AE peak amplitude and energy parameter were fed in to neural network (NN) for predicting the tensile strength of carbon/epoxy composite laminates [14, 15]. Arumugam et al [16] predicted residual tensile strength of impacted carbon/epoxy laminates using ANN with $\mathrm{AE}$ as input cumulative counts and amplitude frequency data collected up to $50 \%$ of failure loads.

ANN models would be able to capture more of the non-linear characteristics and work fairly well with linear cumulative damage rule [17]. The similar NN model was used to detect an embedded delamination size, shape and location in FRP composite laminates using natural 
frequencies as input parameter. The actual efficiency of NN model was found better when it was trained and tested with real life data [18]. The training and testing results in the NN models have shown that they have strong potential for predicting the split tensile strength and percentage of water absorption values of concretes [19]. Recent studies have proved that ANN drastically reduced the computational cost for large composite structures while providing sufficient accuracy [20]. ANN model was developed for computing transverse elasticity modulus of unidirectional composites structure [21]. Shao et al [22] used fuzzy neural network to provide desirable tolerance, and improve identification accuracy in structural damage detection. Adaptive neuro-fuzzy inference system (ANFIS) and multilayer feed forward ANN learning by back propagation algorithm can be used as a non-destructive procedure for health monitoring of structural elements [23].

Zhang and Friedrich [24] proposed ANN models for predicting fatigue life, wear performance and dynamic properties of polymer composite materials. ANN could be used to accurately predict additional material properties such as heat transfer coefficients and crack speed [25]. It is found that once the neural network training is completed, the optimum values for not only learned thermal conditions but also for unlearned thermal conditions can be predicted very quickly by making use of the neural network [26]. Bayesian probabilistic neural network (BPNN) was used to detect the damage in sandwich composite materials identifying the type, location and extent of the damage [27]. The success of ANN training algorithms depends on the training data set and the structure of the network [28]. The NN proved to be more consistent with its lower standard deviation for the prediction of the shear strength of FRP reinforced concrete members [29]. Topcu has successfully developed ANN model for prediction of compressive strength and splitting tensile strength of concrete structure [30].

In the past, many researchers have revealed the capability of ANN models for predicting the mechanical strength and properties in composite materials. But there is a lack of research on developing the ANN model with high prediction accuracy using acoustic emission (AE) parameters. This research focuses on improving prediction accuracy and rate of convergence of NN by radial basis function (RBF) and generalised regression (GR) techniques. Hence, multilayer feed forward type ANN learning algorithms were used to develop the radial basis function neural network (RBFNN) model and generalised regression neural network (GRNN) model from AE parameters. However, durability of the glass fiber reinforced composite laminates in seawater environment has hardly been investigated using 
AE parameters with the help of RBFNN model and GRNN model. The prediction ability of GRNN model is very satisfactory, after the desired training set selection and spread optimization [31].

In the present work, degradation behavior of glass/epoxy composite laminates exposed to seawater environment was investigated using acoustic emission (AE). The acoustic activity and acoustic signatures of aged glass/epoxy composite laminates were recorded during 3 point bending test with $\mathrm{AE}$ monitoring. The significant AE parameters such as $\mathrm{AE}$ hits, cumulative $\mathrm{AE}$ counts, cumulative $\mathrm{AE}$ energy, cumulative $\mathrm{AE}$ signal strength and cumulative AE absolute energy were considered as training data input. The radial basis function neural network (RBFNN) model and generalised regression neural network (GRNN) model was developed from $40 \%$ to $70 \%$ of failure load of AE parameters as training data input. RBFNN model was able to predict the ultimate failure strength and validated with the experimental results with the percentage error well within $0.5 \%-7.2 \%$ whereas GRNN model was able to predict the ultimate failure strength with the percentage error well within $0.5 \%-4.4 \%$. This model can be used for health monitoring of composite materials for application in the shipping industry.

\section{Experimental Procedure}

\subsection{Materials and specimen preparation}

Laminates were fabricated from 16 layer symmetrical bidirectional plies of E-Glass fabric (300 GSM) impregnated in epoxy resin (LY556) with hardener (HY 951) by sealantvacuum bagging at 1 atmospheric pressure for 2 hours, curing at room temperature for 8 hours and subsequent post-curing processing at $100^{\circ} \mathrm{C}$ for 2 hours. The glass/epoxy laminates were built up to a total thickness of $3.4( \pm 0.2) \mathrm{mm}$. The specimens were cut from the laminates using water jet cutting as per ASTM D790 for flexural testing. The dimensions of the specimen are kept as $120 \mathrm{~mm} \times 20 \mathrm{~mm}$.

\subsection{Exposure to seawater environment}

The specimens were immersed in a container with sea water at room temperature for various periods as shown in Fig.1, and regular weight gain measurements were carried out. Water uptake was calculated as weight gains related to the weight of the dry specimen. Four composite specimens were tested and results averaged. 


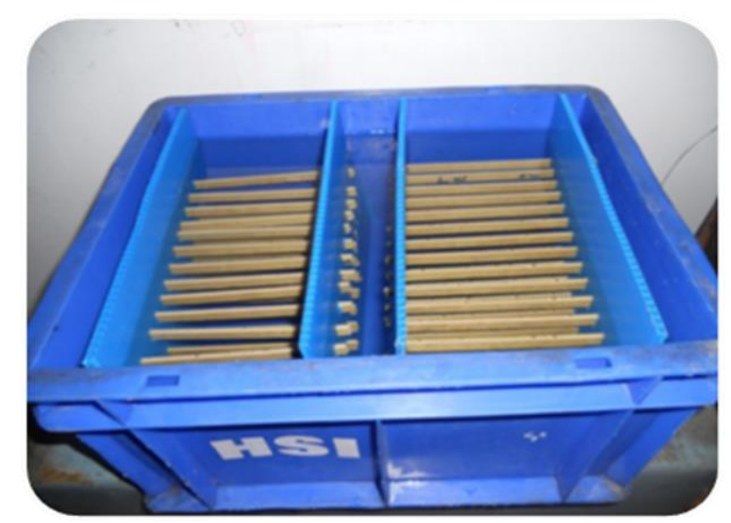

Fig.1: GFRP specimens immersed in seawater environment

\subsection{Flexural testing}

Three points bending testing was carried out using a 100kN Tinius Olsen universal testing machine to compute the mechanical properties as shown in Fig.2. A span-to-depth ratio equal to 25 has been used, so that span was finally equal to $85 \mathrm{~mm}$. The cross-head speed for testing of all the specimens was kept at $0.25 \mathrm{~mm} / \mathrm{min}$.

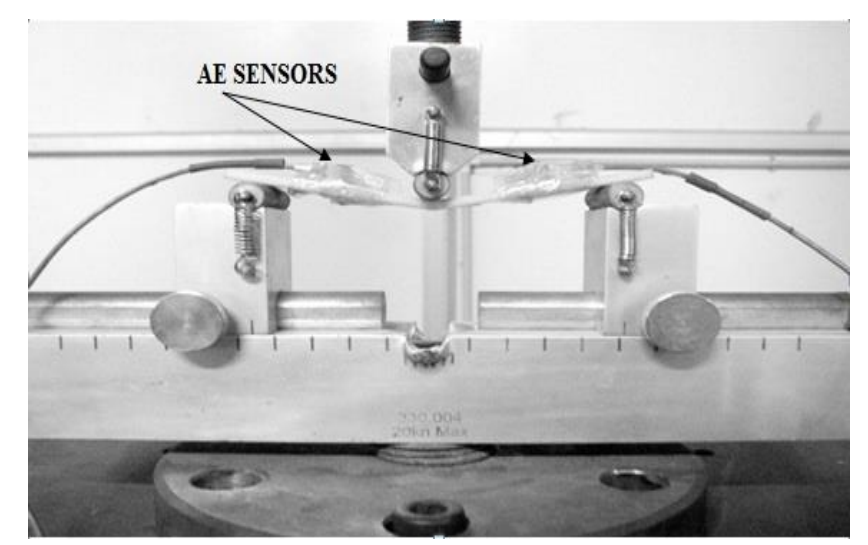

Fig.2: Specimen under flexural loading with AE sensors

\subsection{Acoustic emission monitoring}

An 8 channel AE system supplied by physical acoustics corporation (PAC) with a sampling rate of $3 \mathrm{MHz}$ and $40 \mathrm{~dB}$ pre-amplification was used for this study. Preamplifiers having a bandwidth of $10 \mathrm{kHz}-2 \mathrm{MHz}$ are used. The ambient noise was filtered using a threshold of $45 \mathrm{~dB}$. AE measurements were performed using two wide-band sensors at a mutual distance of $50 \mathrm{~mm}$. High vacuum silicon grease was used as a couplant between specimens' surface and AE sensors. Pencil lead break procedure was used to generate repeatable AE signals for the calibration of each sensor. The wave velocity of GFRP 
composite laminates was found to be $3127 \mathrm{~m} / \mathrm{s}$. The input parameters used for AE monitoring are as follows: peak definition time $(\mathrm{PDT})=25 \mu \mathrm{s}$, hit definition time $(\mathrm{HDT})=160 \mu \mathrm{s}$, hit lock-out time $(\mathrm{HLT})=300 \mu \mathrm{s}$. The significant AE parameters such as counts, energy, signal strength, absolute energy, and hits were recorded for all the specimens.

\section{Artificial Neural Networks}

ANN resembles the model of the biological neural system of human beings. It models the most important feature of the brain, the ability to learn. An ANN is defined by three types of parameters:

a. The interconnection pattern between the different layers of neurons

b. The learning process to update the weights of the interconnections

c. The activation function to convert a neuron's weighted input to its output activation.

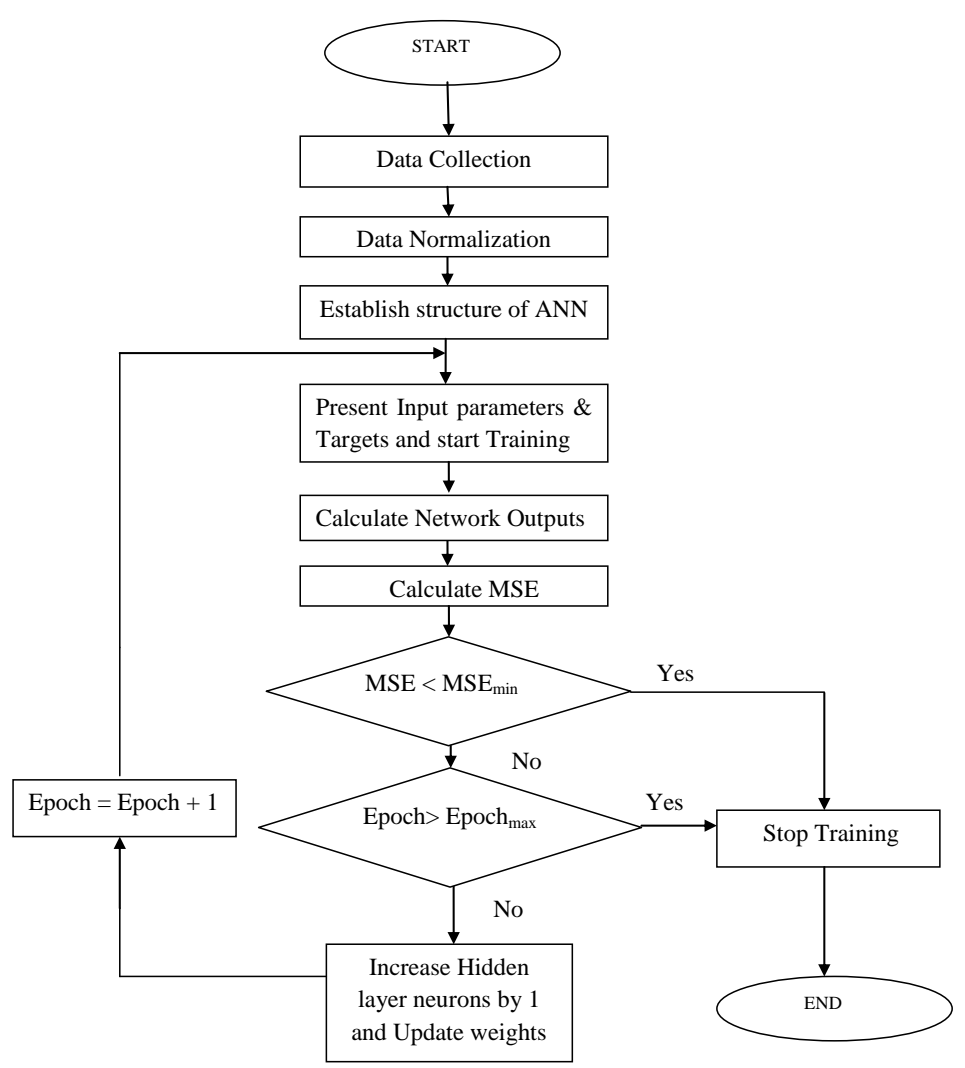

Fig.3: Schematic representation of ANN training process

Fig.3 shows the schematic representation for ANN modelling steps in training the neural network until the specified mean square error and epochs are reached. The proposed 
methodology uses two different types of artificial neural networks, namely radial basis function neural networks (RBFNN) and generalised regression neural networks (GRNN).

\subsection{Radial Basis Function Neural Network (RBFNN)}

Radial basis function network is a type of ANN which is used in problems of regression, classification and prediction. It is a real valued function whose value depends on the distance from its receptive field centre $\chi$ to the input $\mathrm{x}$. It is a positive radially symmetric function whose centre has separate maximum and the value drops to zero away from the centre. If the distance between $\mathrm{x}$ and $\chi$ is smaller than the receptive field width $\mu$, the function is said to be appreciable.

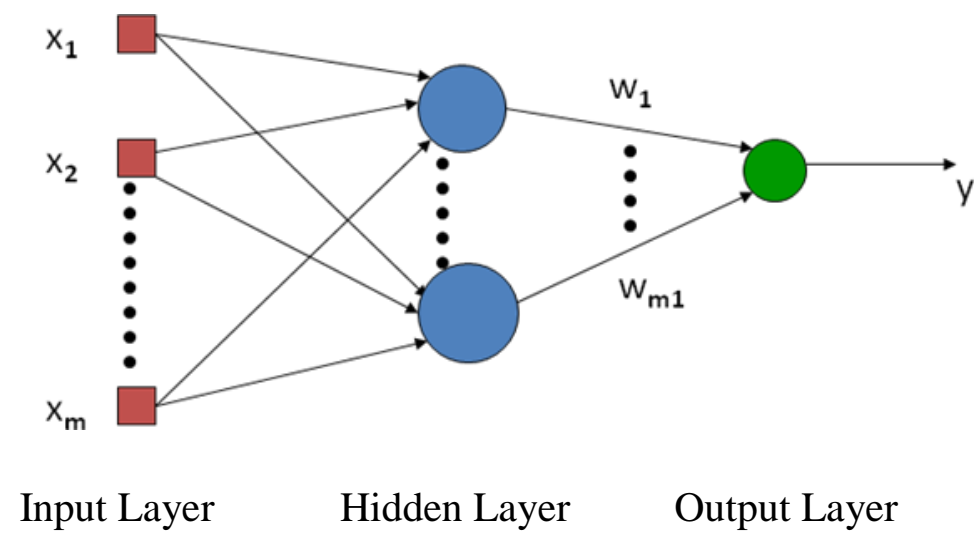

Fig.4: Architecture of RBF network

A radial basis function is one whose output depends on the distance of the input from a given stored vector. In a RBF network, the hidden layer uses neurons with RBF activation functions which describe local receptors. The outputs of the hidden neurons are combined linearly with one output node. Typical architecture of RBF network is shown in Fig.4. It consists of one hidden layer which uses RBF activation function $\varphi_{1} \ldots \varphi_{m 1}$ and output layer with linear activation function.

$$
y=w_{1} \varphi_{1}\left(\left\|x-t_{1}\right\|\right)+\ldots+w_{m 1} \varphi_{m 1}\left(\left\|x-t_{m 1}\right\|\right)
$$

where $\|x-t\|$ is distance of $x=\left(x_{1}, \ldots, x_{m}\right)$ from vector $\mathrm{t}$

Typically, RBF has three layers feed-forward structures. The first layer, which serves as an input to the hidden layer by giving inputs without changing their values, is called input layer. The second layer, where all the neurons simultaneously receive the n-dimensional 
real-valued input vector $\mathrm{x}$, is called hidden layer. Each neuron in this layer uses RBF as the activation function. A commonly used RBF is Gaussian basis function.

$$
R_{j}(x)=\exp \left[\frac{-\left\|x-\chi_{j}\right\|^{2}}{2 \sigma_{j}^{2}}\right]
$$

where $\chi_{j}$ and $\sigma_{j}$ are the mean and the standard deviation of the receptive field of the $\mathrm{j}^{\text {th }}$ hidden layer neuron and $\mathrm{R}_{\mathrm{j}}(\mathrm{x})$ is the corresponding activation function. The third layer is the output layer. The output can be expressed as $\mathrm{y}=\left[\mathrm{y}_{1}, \mathrm{y}_{2}, \mathrm{y}_{3} \ldots \ldots . ., \mathrm{y}_{\mathrm{k}}\right]$ with $\mathrm{y}_{\mathrm{i}}$ as the output of the $\mathrm{i}^{\text {th }}$ neuron given by

$$
y_{i}=\sum_{j=1}^{h} w_{j i} R_{j}(x) \quad \text { for } \mathrm{i}=1,2,3 \ldots . \mathrm{k}
$$

where $\mathrm{h}$ is the number of neurons in the hidden layer and $\mathrm{w}_{\mathrm{ji}}$ is the weight associated with the $\mathrm{j}^{\text {th }}$ hidden layer neuron and $\mathrm{i}^{\text {th }}$ output layer neuron.

\subsection{Generalised Regression Neural Network (GRNN)}

The GRNN is a one-pass learning algorithm which has a highly parallel structure. Although, with sparse data in a multidimensional measurement space, smooth transition is provided by the algorithm from one observed value to another, the algorithmic form is used for any regression problem in which an assumption of linearity is not justified.

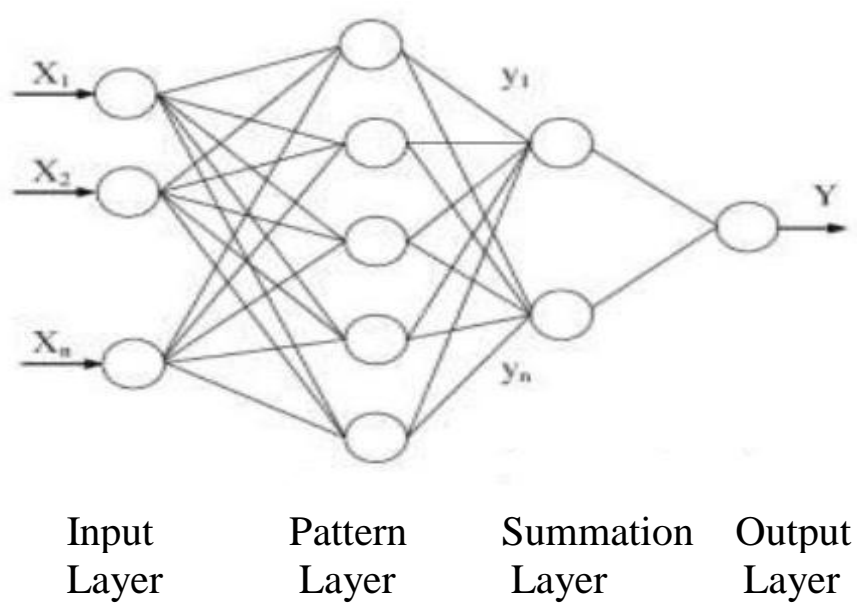

Fig.5: Architecture of GRNN network

A GRNN is a variation of the radial basis neural network, which is based on kernel regression networks. An iterative training procedure is not required in GRNN unlike 
multilayer perceptron based back propagation (BP) networks. It approximates any arbitrary function between input and output vectors by drawing the function estimate directly from the training data. A GRNN consists of four layers with input layer, pattern layer, summation layer and output layer, as shown in Fig.5. The number of input units in the input layer depends on the total number of the observation parameters. The first layer is connected to the pattern layer and each neuron presents a training pattern and its output in this layer. The pattern layer is being connected to the summation layer. There are two different types of summation units present in summation layer, which are the single division unit and summation units.

During training of network, radial basis and linear activation functions are used in hidden and output layers respectively. Each pattern layer unit is connected to the two neurons in the summation layer named as S and D neurons. S summation neuron is used to compute the sum of weighted responses of the pattern layer. D summation neuron calculates un-weighted outputs of pattern neurons. The output layer divides the output of each Ssummation neuron by that of each D-summation neuron which yields the predicted value $\mathrm{Y}_{\mathrm{i}}$ to an unknown input vector $\mathrm{x}$ as given in equation 4, 5 as follows.

$$
Y_{i}^{\prime}=\frac{\sum_{i=1}^{n} y_{i} \exp \left(-D\left(x, x_{i}\right)\right)}{\sum_{i=1}^{n} \exp \left(-D\left(x, x_{i}\right)\right)}
$$

where,

$$
D\left(x, x_{i}\right)=\sum_{k=1}^{m}\left(\frac{x_{i}-x_{i k}}{\sigma}\right)^{2}
$$

where $\mathrm{y}_{\mathrm{i}}$ is the weight connection between the $\mathrm{i}^{\text {th }}$ neuron in the pattern layer and the $\mathrm{S}$ is summation neuron, $\mathrm{n}$ indicates the number of the training patterns, $\mathrm{D}$ denotes the Gaussian function, $\mathrm{m}$ is the number of elements of an input vector, $\mathrm{x}_{\mathrm{k}}$ and $\mathrm{x}_{\mathrm{ik}}$ are the $\mathrm{j}^{\text {th }}$ element of $\mathrm{x}$ and $\mathrm{x}_{\mathrm{i}}$, respectively and $\mathrm{r}$ is the spread parameter of the Gaussian function.

\section{Results and Discussion}

The Glass-epoxy composite laminates exposed to seawater in the period of 4,5,6,7 months are subjected to flexural testing with AE monitoring. Acoustic emission data are collected during loading until the failure of the specimens. The ANN prediction models were developed from the recorded $\mathrm{AE}$ data during testing. The feature which was followed for developing models can be summarized as follows: 
- Developed RBFNN and GRNN models have higher accuracy and quicker convergence when compared with other supervised learning network.

- The optimization of spread value $\sigma$ in RBFNN as 0.108 and GRNN as 0.01 .

- The normalized AE cumulative parameters are chosen as input for training network.

- The input AE parameters are considered only in the linear region (500 ms to $800 \mathrm{~ms}$ ) of every $1 \%$ of ultimate load ( $40 \%$ to $70 \%$ ) for minimising the error.

- The mean and median of predicted failure load is also computed and compared with experimental failure load.

\subsection{Arrangement and Normalization of AE data}

The acquired AE parameters are chosen as input data and are considered cumulatively for every $1 \%$ of load. The five considered $\mathrm{AE}$ parameters are number of hits, cumulative counts, cumulative energy, cumulative absolute energy and cumulative signal strength. The Target parameter used is maximum failure load of each specimen. All the data used as inputs and targets are normalized individually between -1 and +1 and are given to network for training. There are totally 20 composite laminates taken for experimentation. These specimens are of different aged types of 4,5,6,7 months with each aged type consisting of five specimens. Out of these 20 specimens, 16 specimens ( 4 in each type) are used for training the network and 4 specimens ( 1 in each type) are used for testing.

\subsection{Selection of Range of Input Parameters for Training}

The range of input parameters to be selected for training the network is based on the analysis of variation of \% load versus all the five input parameters. \% Load versus all of the input parameters plotted for each aged category of specimens is shown in fig.6 to fig.9.
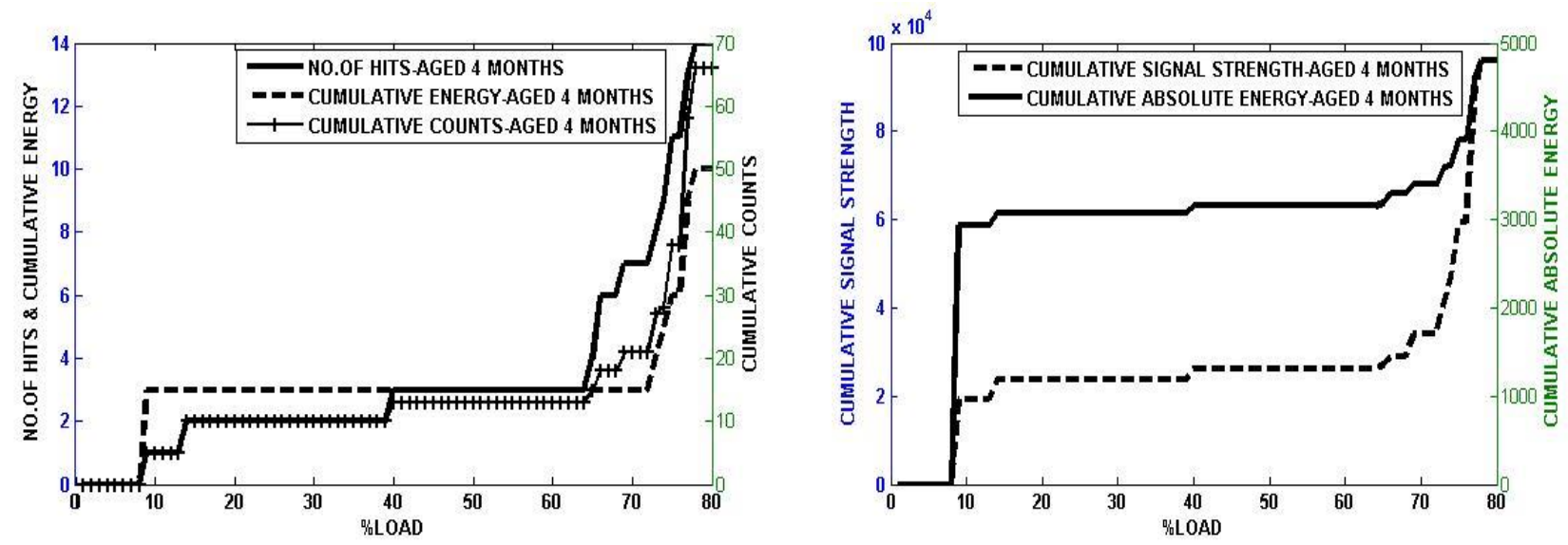

Fig.6: Input parameters Vs various \% of load for aged-4 months category specimen 

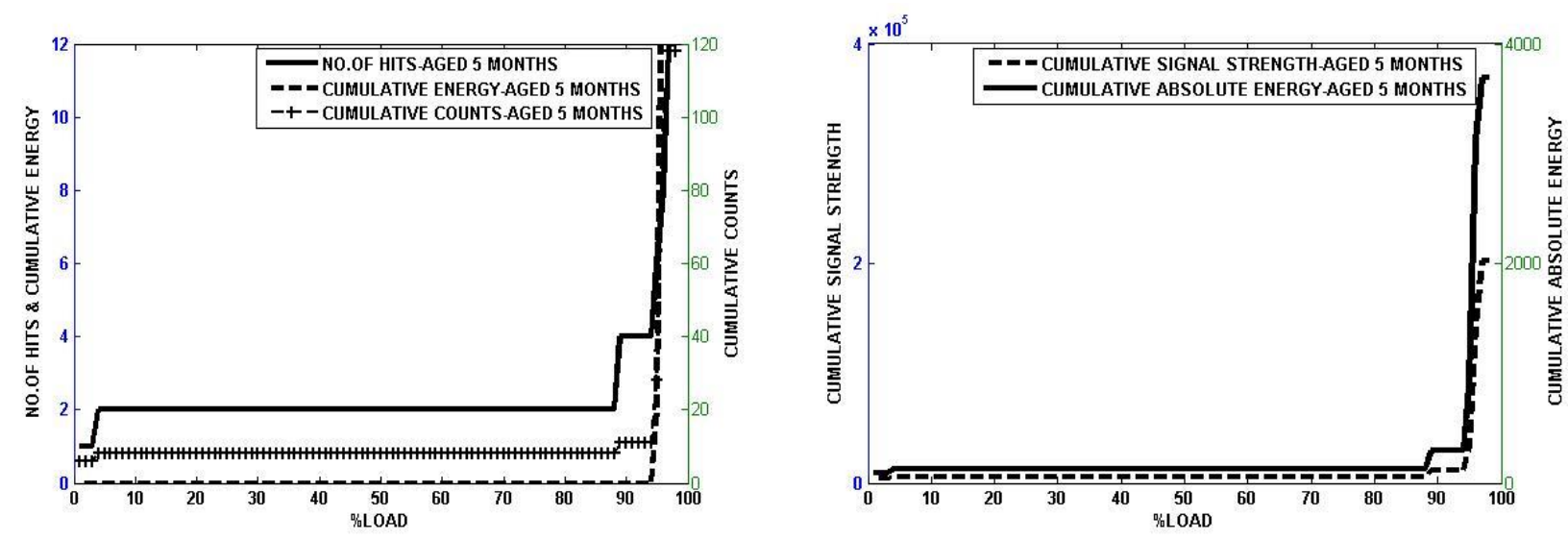

Fig.7: Input parameters Vs various $\%$ of load for aged-5 months category specimen
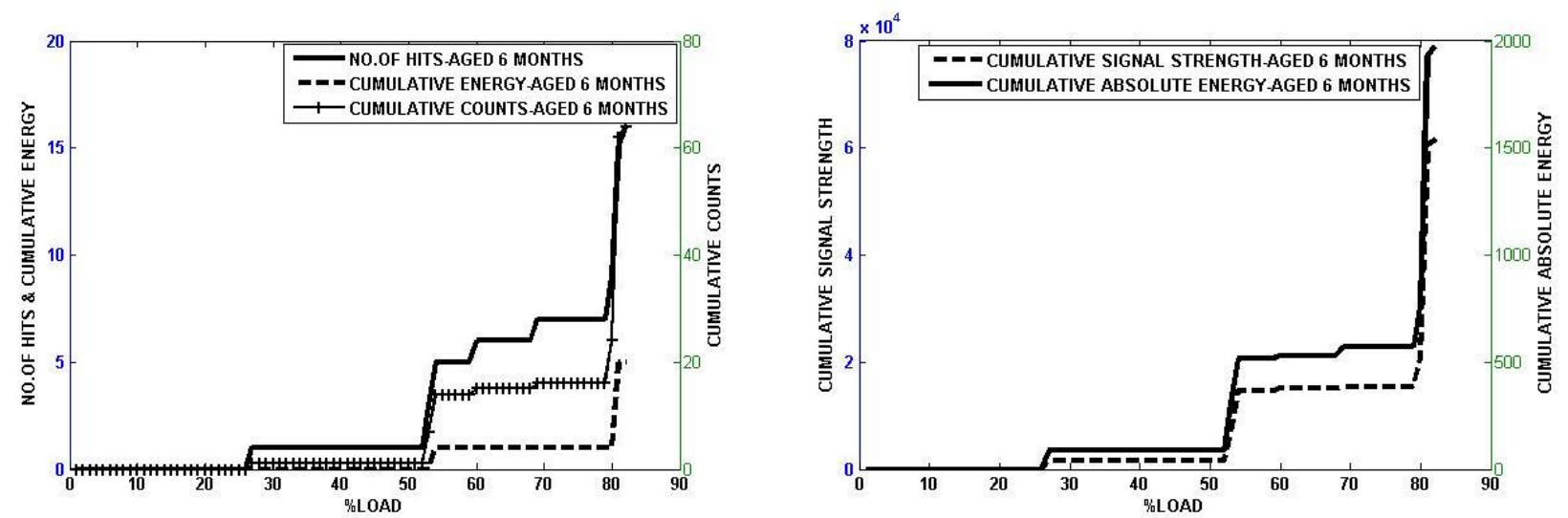

Fig.8: Input parameters Vs various \% of load for aged-6 months category specimen
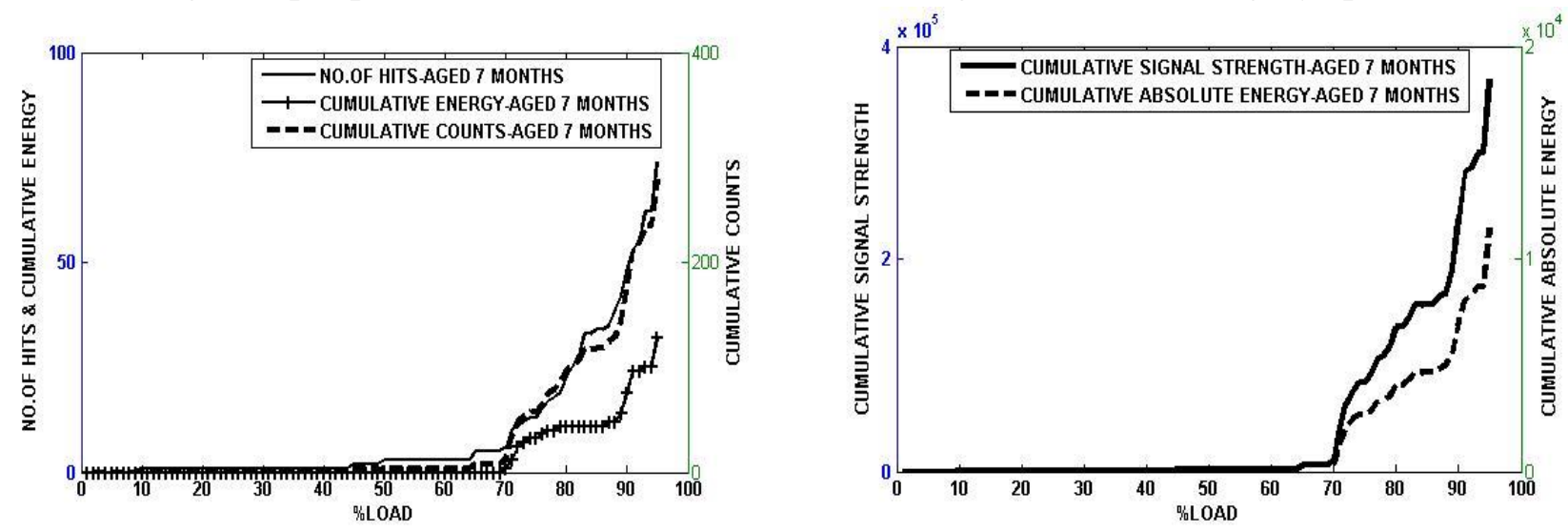

Fig.9: Input parameters Vs various \% of load for aged-7 months category specimen

From the above Fig.6 to Fig.9, it is clear that all the input parameters are almost in the constant range when the load varies from $40 \%$ to $70 \%$. At below $40 \%$ of load, some of the specimens have no hits occurring in them and at above $70 \%$ of load, input parameters are increasing and varying drastically to a very high value. This may be attributed to echo of failure on the specimen at the time of increasing damage in it at higher loads [11, 12, 32]. So input parameters used for training are considered from $40 \%$ to $70 \%$ load for least error. 


\subsection{Prediction with RBFNN and GRNN Models}

\subsubsection{Developing the RBFNN and GRNN Models}

The failure load prediction is done with RBFNN and GRNN models since the training is easier and faster with higher accuracy and quicker convergence when compared to the back propagation (BP) network model. The coefficients of GRNN model are determined in one pass through the data and hence no iterative algorithm is required when compared to the BP network model and RBFNN model. Both of the network models have feed forward type architecture and uses supervised learning where the target parameter of ultimate failure load of each specimen is specified during training. The activation function used is the Gaussian distribution function where the tuning parameter is the spread value. The spread value $\sigma$ is found optimally as 0.108 in RBFNN model and 0.01 in GRNN model. The number of neurons in input layer is 5 since there are 5 input parameters used for training and the number of neurons in the output layer is 1 since there is only one target used for training. The number of neurons in the hidden layer in RBFNN model is found optimally as 23 by iteratively increasing till it reaches the specified mean square error (MSE) of 0.01 .

Fig.10 (a) shows the structure of the RBFNN model with 5 neurons in the input layer, 23 neurons in the hidden layer and 1 neuron in the output layer. From fig.10(b) and fig.10(d) shows the regression plot of RBFNN and GRNN models with the correlation coefficient, R as 0.99233 and 0.99647 respectively which indicates that both the models are well trained such that the MSE between network outputs and targets reached the specified minimum value of 0.01. Fig.10(c) shows the structure of the GRNN model with 5 neurons in the input layer, 39 neurons in the hidden layer and 1 neuron in the output layer. Fig.10 (e) shows the training performance of RBFNN model where the MSE of 0.0079 is reached at 23 epochs.

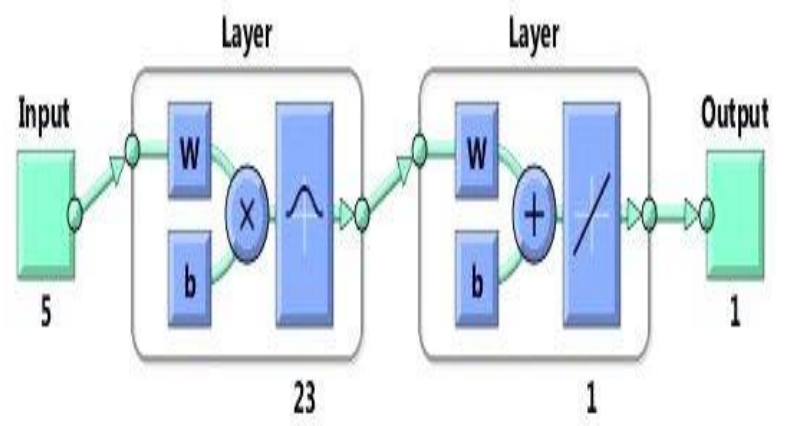

(a)

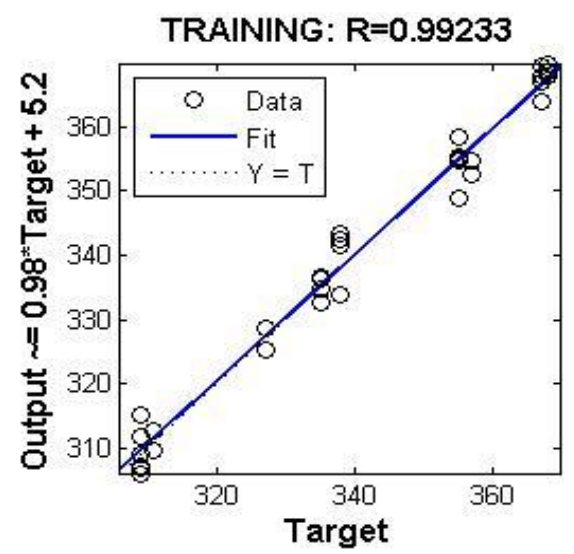

(b) 


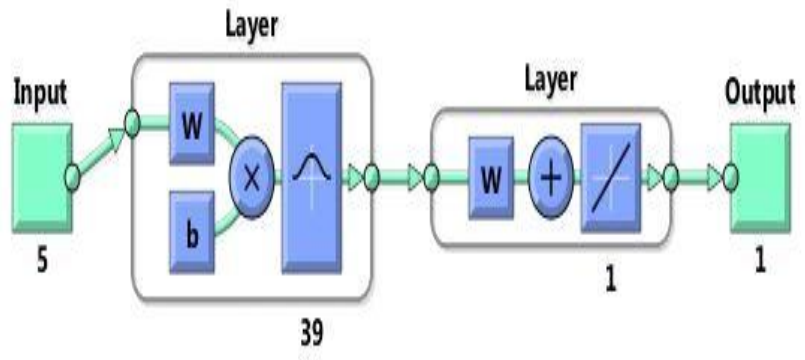

(c)
TRAINING: $\mathrm{R}=0.99647$

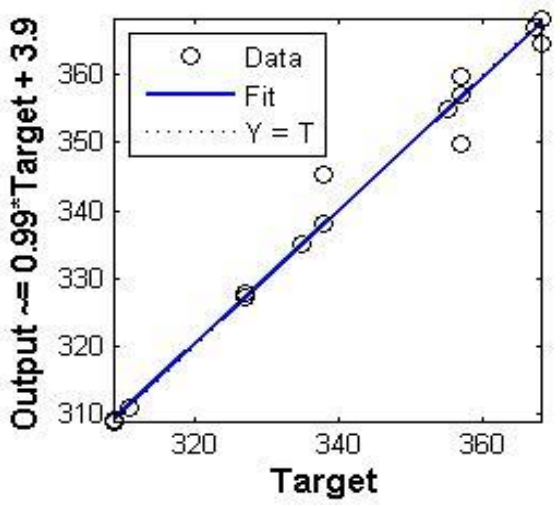

(d)

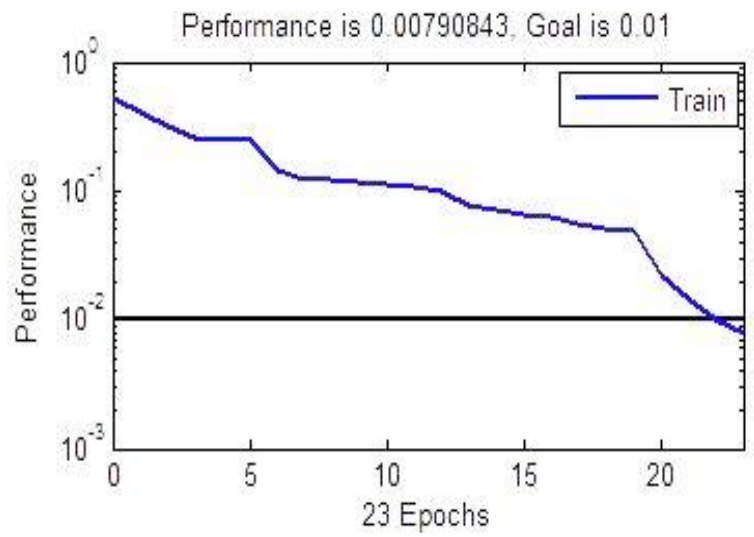

(e)

Fig.10 (a) Structure of RBFNN model (b) Regression plot of RBFNN training (c) Structure of GRNN model (d) Regression plot of GRNN training (e) Training performance of RBFNN model

\subsubsection{Simulation and Testing the RBFNN and GRNN Models}

Four new specimens that are not used in training the network are used for testing. The same five input parameters are calculated cumulatively for each new specimen in time scale of every $1 \mathrm{~ms}$ and are given as testing inputs to the network. The specimen is tested from 1 $\mathrm{ms}$ time to a maximum of $800 \mathrm{~ms}$ (where original breaking time is around from $1400 \mathrm{~ms}$ to $1600 \mathrm{~ms}$ identified from trained specimen data) up to which the specimen is not overly stressed. To identify the time range at which the trained network predicts the failure load of a new specimen with minimum error, trained specimens are simulated using the trained network and it is found that from $500 \mathrm{~ms}$ to $800 \mathrm{~ms}$, RBFNN and GRNN models predict with minimum error. 


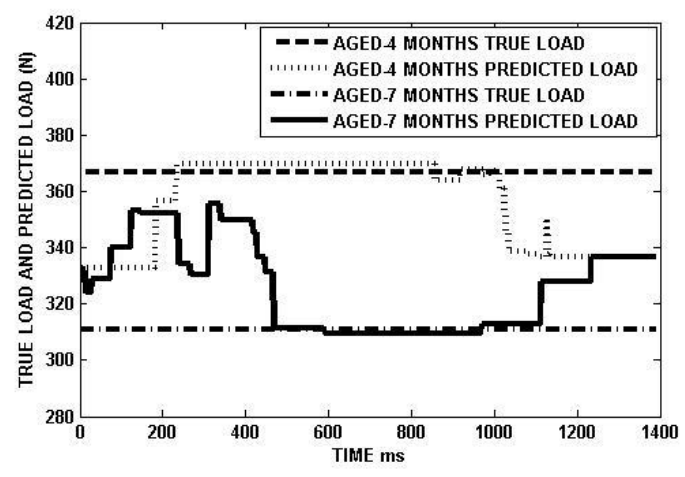

(a)

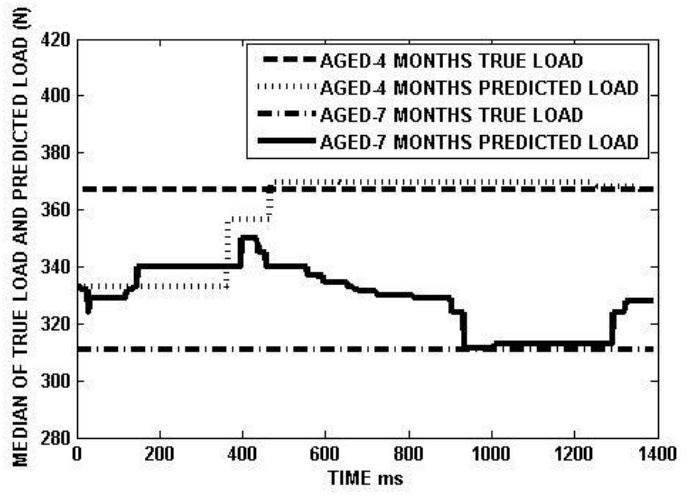

(b)

Fig.11: Time versus (a) True load and predicted load (b) Median of true load and predicted load of trained specimens using RBFNN Model

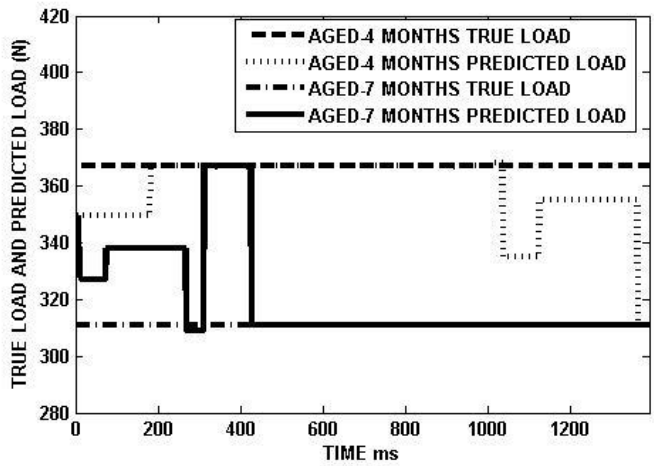

(a)

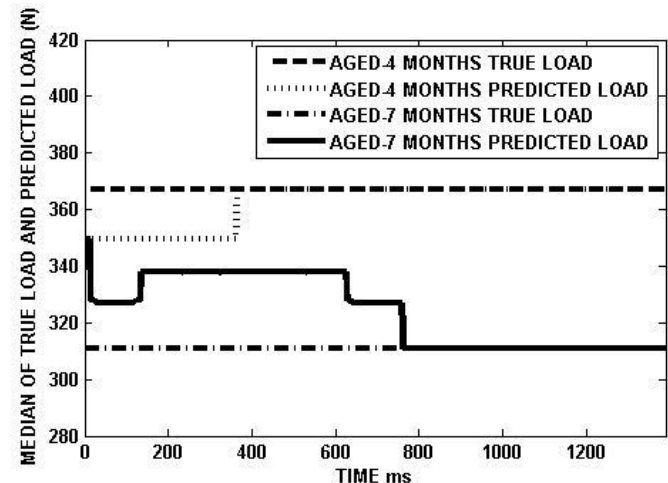

(b)

Fig.12: Time versus (a) True load and predicted load (b) Median of true load and predicted load of trained specimens using GRNN Model

Fig.11 and Fig.12 show the plot of time versus true load and predicted load and with cumulative median of true load and predicted load with RBFNN and GRNN models respectively, for trained specimens used in simulation to identify the time range at which the trained RBFNN and GRNN model predicts the failure load of a new specimen with minimum MSE of 0.0079 .

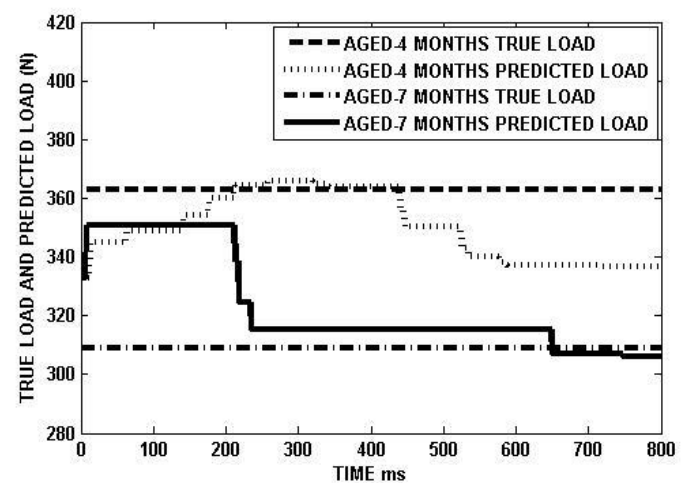

(a)

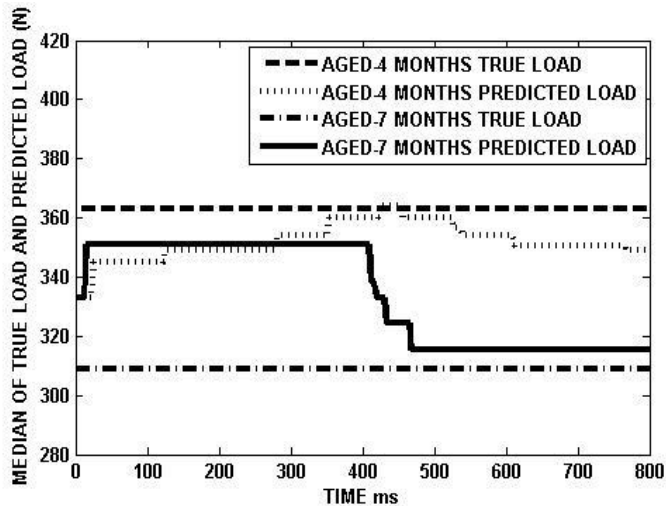

(b)

Fig.13: Time versus (a) True load and predicted load (b) Median of true load and predicted load of new testing specimens using RBFNN model 


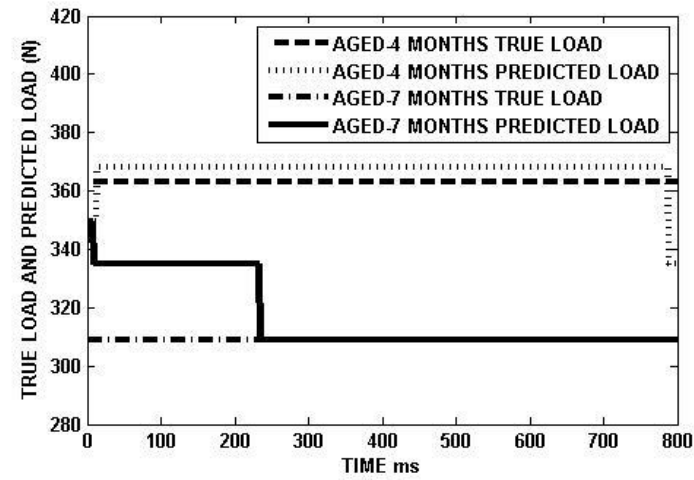

(a)

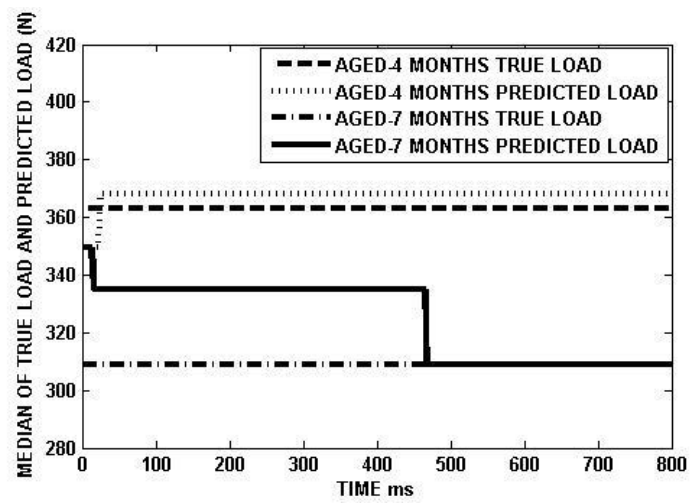

(b)

Fig.14 Time versus (a) True load and predicted load (b) Median of true load and predicted load of new testing specimens using GRNN model

The load is applied to the four new specimens that are not used in training and they are tested using the trained RBFNN and GRNN models and hence mean of predicted failure load and median of predicted failure load are found out between $500 \mathrm{~ms}$ and $800 \mathrm{~ms}$ from the results of tested specimens. Evidently, Figs.13 and 14 show the plot of time versus true load and predicted load and with cumulative median of true load and predicted load, for validation of developed RBFNN and GRNN models respectively. Mean and median of their predicted failure load values are plotted against experimental failure load for various ageing categories as shown in fig.15 (a) and fig.16 (a) for RBFNN and GRNN models respectively.

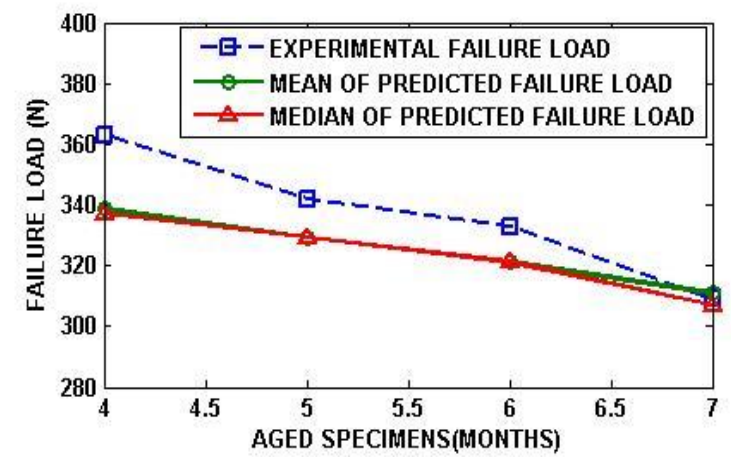

Fig.15 (a): Variation of ageing with ultimate strength prediction with RBFNN model

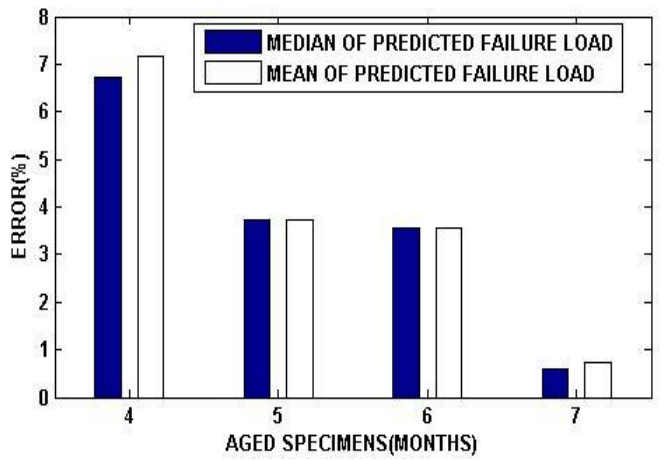

Fig. 15 (b): Variation of \% error in aged specimens with RBFNN model 


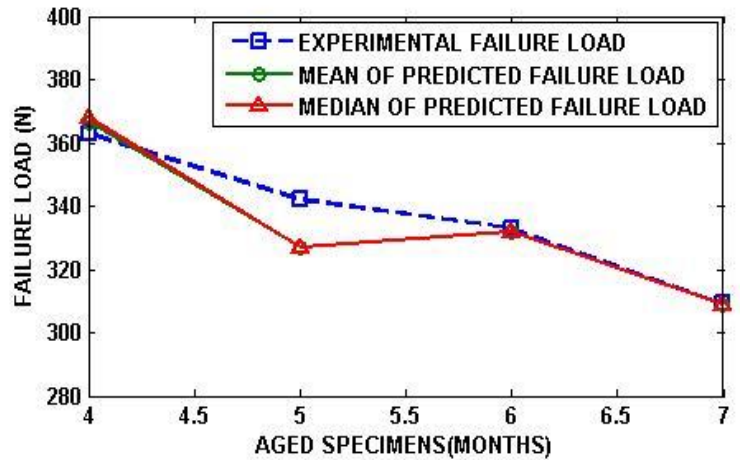

Fig.16 (a) Variation of ageing with ultimate strength prediction with GRNN model

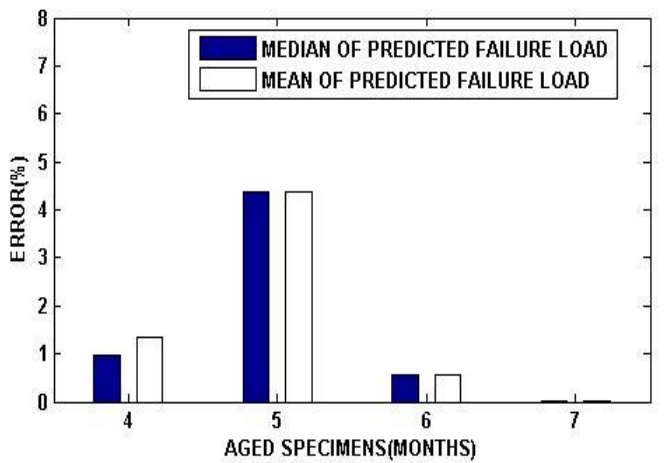

Fig.16 (b) Variation of \% error in aged specimens with GRNN model

Fig.15 (a) and Fig.15 (b) show the variation of ageing with ultimate failure strength with experimental results and by using RBFNN and GRNN model prediction respectively. It is found that when ageing increases, ultimate strength starts decreasing. Fig.16 (a) and Fig.16 (b) shows the plot of prediction error in failure load with RBFNN and GRNN model respectively during testing of new specimens. It is found that from the experimental and predicted values of failure load, the percentage error is well within $0.5 \%-7.2 \%$ using RBFNN model and $0.5 \%-4.4 \%$ using GRNN model. It is also found that median is the best parameter when compared with mean, with less error.

\section{Conclusions}

The ultimate flexural strength of GFRP composite laminates was decreased with increasing the period of 4, 5, 6 and 7 month in seawater treatment. The degradation effects of seawater in GFRP composite specimens were analysed using acoustic emission monitoring. AE hits, cumulative counts, cumulative energy, cumulative signal strength and cumulative absolute energy were computed in the range of $40 \%$ to $70 \%$ of the maximum failure load and they were used as input data to ANN for developing the model. The radial basis function neural network (RBFNN) and generalised regression neural network (GRNN) models were developed to predict the failure strength of GFRP composite specimens. The variation of the experimental and predicted failure strength of RBFNN and GRNN models were determined with an error tolerance of within $0.5 \%-7.2 \%$ and $0.5 \%-4.4 \%$ respectively. The prediction accuracy of the GRNN model is found to be better than the RBFNN model. Therefore, AE combined with the ANN becomes an effective method of health monitoring of composite materials for application in the shipping industry. 


\section{Acknowledgements}

The support from Anna Centenary research fellowship, Anna University Chennai, Tamilnadu in India, is gratefully acknowledged for providing research fellowship and also the authors would like to thank for the extended support from Advanced Polymer and Composites (APC) Research Group, School of Engineering, University of Portsmouth, Anglesea Road, Anglesea Building, Portsmouth, Hampshire PO1 3DJ, UK.

\section{References}

[1]. Shan Y, Liao K. Environmental fatigue of unidirectional glass-carbon fiber reinforced hybrid composite. Compos Part B 2001; 32: 355-363.

[2]. Thomas KT, Scott K, Kyejune Le, Jason B, Jack B. Aging of polymeric composite specimens for $5000 \mathrm{~h}$ at elevated pressure and temperature. Compos Sci Technol $2001 ; 61: 75-86$.

[3]. Allred RE. The effect of temperature and moisture content on the flexural response of kevlar/epoxy laminates II (+ or $-45,0 / 90)$ filament orientation. J Compos Mater 1981; 15:117-132.

[4]. Chateauminois A, Chabert B, Soulier JP, Vincent L. Hygrothermal ageing effects on the static fatigue of glass/epoxy composites. Compos 1993; 24(7):547-555.

[5]. Gautier L, Mortaigne B, Bellenger V. Interface damage study of hydrothermally aged glass-fiber reinforced polyester composites. Compos Sci Technol 1999; 59:23292337.

[6]. Bassir DB, Guessama S, Boubakar L. Hybrid computational strategy based on ANN and GAPS: application for identification of a non-linear model of composite materials. Compos Struct 2009; 88(2):262-270.

[7].Perera R, Barchin M, Arteaga A, De Diego A. Prediction of the ultimate strength of reinforced concrete beams FRP strengthened in shear using neural networks. Compos Part B 2010; 41:287-298.

[8].Giordano M, Calabro A, Esposito C, Amorec A, Nicolais L. An acoustic emission characterization of the failure modes in polymer composite materials. Compos Sci Technol 1998; 58:1923-1928.

[9]. American society for testing materials, ASTM E1316, standard terminology for nondestructive examinations. In: Annual book of ASTM standards, non-destructive testing, vol. 03. Philadelphia: ASTM. 
[10].Barre S, Benzeggagh ML. On the use of acoustic emission to investigate damage mechanism in glass-fiber reinforced polypropylene. Compos Sci Technol 1994; 52:369-376.

[11].Arumugam V, Suresh Kumar C, Santulli C, Sarasini F, Joseph Stanley A. A global method for the identification of failure modes in fiber glass using acoustic Emission. $\mathbf{J}$ Test and Eval 2011; 39(5):103730

[12].Arumugam V, Suresh Kumar C, Santulli C, Sarasini F, Joseph Stanley A. Identification of failure modes in composites from clustered acoustic emission data using pattern recognition and wavelet transformation, Arab J Sci Eng 2013 38:10871102

[13].Bar HN, Bhat MR, Murthy CRL. Parametric analysis of acoustic emission signals for evaluating damage in composites using PVDF film sensors. NDT Inter 2005; 24:121134.

[14].Walker JL, Hill EVK. Back propagation neural network for predicting ultimate strength of unidirectional graphite/epoxy tensile specimens. Adv Perform mater 1996; $3: 75-83$.

[15].Sasikumar T, Rajendraboopathy S, Usha KM, Vasudev ES, Failure strength prediction of unidirectional tensile coupons using acoustic emission peak amplitude and energy parameter with artificial neural networks. Compos Sci. Technol 2009; 69:1151-1155.

[16].Arumugam V, Naren Shankar R, Sridhar BTN, Joseph Stanley A, Ultimate strength prediction of carbon/epoxy tensile specimens from acoustic emission data. J Mater. Sci. Technol 2010; 26(8):725-729.

[17].Sung WC, Eun Jung S, Thomas HH. Prediction of fatigue damage growth in notched composite laminates using an artificial neural network. Compos Sci Technol 2003; 63:661-675.

[18].Chakraborty D. Artificial neural network based delamination prediction in laminated composites. Mater Des 2004; 26(1):1-7.

[19].Ali Nazir, Shadi Riahi. Prediction split tensile strength and water permeability of high strength concrete containing $\mathrm{TiO}_{2}$ nanoparticles by artificial neural network and genetic programming. Compos Part B 2011; 42:473-488. 
[20].Chiachio M, Chiachio J, Rus G. Reliability in composites - A selective review and survey of current development. Compos Part B 2012; 43:902-913.

[21].Eduardo Cesar BC, Raimundo Carlos SF Jr. Using neural networks to modeling the transverse elasticity modulus of unidirectional composites. Compos Part B 2011; 42:2024-2029.

[22].Shao FJ, Chun MZ, Shuai Z. Two stage structural damage detection using fuzzy neural networks and data fusion techniques. Exp Syst Appl 2011; 38(1):511-519.

[23]. Mahmut Bilgehan. Comparison of ANFIS and NN models - With a study in critical buckling load estimation. Appl. Soft Comput 2011: 11(4):3779-3791.

[24].Zhang Z, Friedrich K. Artificial neural networks applied to polymer composites: a review. Compos Sci Technol 2003; 63:2029-2044.

[25].Peter AL, Richard PD. An artificial neural network approach to multiphase continua constitutive modeling. Compos Part B 2007; 38:817-823.

[26].OotaoY, Tanigawa Y, Nakamura T. Optimization of material composition of FGM hollow circular cylinder under thermal loading: a neural network approach. Compos Part B 1999; 30:415-422.

[27].Frederick JA, David S, Basir S, Andres C. Neural network based non-destructive evaluation of sandwich composites. Compos Part B 2008; 39:217-225.

[28]. Ahmet RB, Murat O, Ilker BT. Using ANN and ANFIS to predict the mechanical and chloride permeability properties of concrete containing GGBFS and CNI. Compos Part B 2013; 45:688-696.

[29].Rizwan B, Ashraf A, Neural network modelling for shear strength of concrete members reinforced with FRP bars. Compos Part B 2012; 43: 3198-3207.

[30].Ilker BT, Mustafa S. Prediction of mechanical properties of recycled aggregate concretes containing silica fume using artificial neural networks and fuzzy logic. Comp Matr Sci 2008; 42(1):74-82.

[31].Jianli L, Wei B, Lei S, Baoqi Z, Weidong G. General regression neural network for prediction of sound absorption coefficients of sandwich structure nonwoven absorbers. Appl Acoust 2014; 76:128-137.

[32].Suresh Kumar C, Arumugam V, Sajith S, Dhakal HN, Risil John. Acoustic emission characterisation of failure modes in hemp/epoxy and glass/epoxy composite laminates. J Nondestruct Eval 2015; 34(4):31. 